\title{
Study of heat transport in a temperature-dependent viscous liquid under temperature modulation
}

\author{
B.S. Bhadauria ${ }^{\mathrm{a}}$ \\ Department of Applied Mathematics, School for Physical Sciences, Babasaheb Bhimrao Ambedkar University, Lucknow- \\ 226025, India.
}

\begin{abstract}
The effect of time-periodic temperature modulation on thermal instability in a temperaturedependent viscous fluid layer has been investigated by performing a weakly nonlinear stability analysis. The amplitude of temperature modulation is considered to be very small, and the disturbances are expanded in terms of power series of amplitude of convection. The Ginzburg-Landau equation for the stationary mode of convection is obtained and consequently the effect of temperature modulation on heat transport has been investigated. Effect of various parameters has been explained graphically. It has been found that, an increment in the values of thermo-rheological parameter and Prandtl number is to enhance the heat transport in the system. Further, temperature modulation can be used to control the heat transport effectively as external mechanism to the system.
\end{abstract}

Keywords: Rayleigh-Bénard convection, Ginzburg Landau equation, Temperature modulation, Temperature dependent viscosity.

\section{Introduction:}

The classical Rayleigh-Bénard convection due to bottom heating is well known and highly explored phenomenon given by Chandrasekhar [1], Drazin and Reid [2]. In most these studies the basic temperature field is considered to be function of space only. However, for real life problems, the basic temperature field has to be a function of both space and time, and this can be used to regulate convection through an external means. Venezian [3], was first to study the effect of temperature modulation on thermal instability in a horizontal fluid layer, as a thermal analogue of Donnely [4], experiments. Using perturbation method and considering free-free surfaces, he calculated the shift in the critical Rayleigh number and showed that the system can be stabilized or destabilized by suitably tuning the frequency of modulation. Later on Rosenblat and Herbert [5], investigated thermal instability for low frequency temperature modulation while Rosenblat and Tanaka [6], Yih and Li [7], and Kumar et al. [8], studied the effect of thermal modulation on the onset of Rayleigh-Bénard convection with rigid boundaries, using Galerkin technique and discussed the stability of the system using Floquet theory. The Venezian problem for free-free surfaces was extended by Finucane and Kelly [9], Roppo et al. [10], for weakly nonlinear thermal instability under temperature modulation. They observed that stable hexagons are produced by the modulation effect near the critical Rayleigh number. Considering various temperature profiles, Bhatia and Bhadauria [11], Bhadauria and Bhatia [12], Bhadauria ([13],[14]) studied temperature modulation of Rayleigh-Bénard convection for rigid-rigid boundaries. Malashetty and Swamy

a e-mail: mathsbsb@yahoo.com
[15], investigated thermal instability of a heated fluid layer subject to both boundary temperature modulation and rotation. They found that the symmetric modulation destabilizes the system at low frequencies but stabilizes at moderate and high frequencies. Asymmetric modulation was shown to stabilize convection for all frequencies. A weakly nonlinear study on thermal instability with temperature modulation using Lorenz model was made by Bhadauria et al. [16], considering various temperature profiles. In addition to finding the effect of temperature modulation, they compared the results of various temperature profiles, in terms of the critical Rayleigh number. Raju and Bhattacharya [17], investigated the onset of thermal instability in a horizontal fluid layer with modulated boundary temperature, and using rigid boundaries.

Most of the above reported studies on temperature modulation are linear. At present there are very few studies available in the literature in which nonlinear analysis has been done under temperature modulation. These are due to Siddheshwar et al. [18], who studied stationary magneto-convection in a Newtonian liquid under temperature or gravity modulation using Ginzburg-Landau model, Bhadauria et al. [19], investigated a non-linear thermal instability in a rotating viscous fluid layer under temperature/gravity modulation, and calculated heat transfer across the fluid layer, Bhadauria et. al, [20] studied weak nonlinear of time-periodic thermal boundary conditions and internal heating on heat transport in a porous medium.

Viscosity is a physical property of fluids. It is the ratio of shear stress to the shear strain. In most of the above studies, the fluid viscosity is considered to be constant. However, in nature, we find a very few examples of fluids possessing this property. In certain situations, it is not necessary that the fluid viscosity is constant. It may vary with distance, temperature or pressure. For example, in coal slurries, the viscosity of the fluid varies with temperature. In general the coefficients of viscosity for real fluids are functions of temperature. In many thermal transport processes, the temperature distribution within the flow field 
is not uniform, i.e., the fluid viscosity may change noticeably if large temperature differences exist in the system. Therefore, it is highly desirable to take into account the temperature dependent viscosity in momentum as well as in the energy equation.

When the fluid viscosity varies with temperature, the top and bottom structures of fluid layer are different, this is known as non-Boussinesq effect (Wu and Libchaber [21]. Kafoussius and Williams, [22], investigated the effect of variable viscosity on the free convection laminar boundary layer flow along a vertical isothermal plate. Kafoussius and Rees [23], examined the effect of temperaturedependent viscosity on the mixed convection laminar boundary layer flow along a vertical isothermal plate. Molla et al. [24], studied the natural convection flow from an isothermal circular cylinder with temperature-dependent viscosity. Pal and Mondal [25], examined the influence of temperaturedependent viscosity and thermal radiation on MHD-forced convection over a non-isothermal wedge. Ching and Cheng [26], studied the temperature-dependent viscosity effects on the natural convection boundary layer on a horizontal elliptical cylinder with constant surface heat flux. Nadeem and Akbar [27], studied the effects of temperature dependent viscosity on the peristaltic flow of a Jeffrey-six constant fluid in a uniform vertical tube. Nield [28], explained an analysis of the extension of the Horton-Rogers-Lapwood problem to the case where the variations of viscosity with temperature is accounted. It was shown that, considering linear stability analysis under Oberbeck-Boussinesq approximation for the case of impermeable, perfectly conducting upper and lower boundaries, the values of the critical value of critical Rayleigh number is $4 \pi^{2}$. However, most of these studies are done with steady temperature gradient across the fluid layer. Recently Bhadauria and Palle Kiran [29] investigated an effect of temperature dependant viscosity on heat transfer in a porous medium by considering weakly non-linear theory.

We see that there is no reported work on non-linear stability that analyze the effect of temperature modulation on temperature-dependent viscous fluid layer. Therefore, in this paper we performed a weakly non-linear analysis of thermal instability in a temperature-dependent viscous fluid layer under temperature modulation and quantify the heat transfer across the fluid layer using amplitude of convection, obtained as the solution of the resultant Ginzburg Landau equation for stationary mode of convection.

\section{Governing Equations}

We consider an infinitely extended horizontal variable viscous fluid layer, confined between two parallel planes which are at $z=0$, lower plane and $z=d$, upper plane. A cartesian frame of reference is chosen in such a way that the origin lies on the lower plane and the $z$-axis in vertically upward direction. The schematic diagrams of the problem for temperature modulation is shown in the Fig.1 respectively. The fluid layer is heated from below and subjected to temperature modulation. The fluid layer is considered to be Boussinesq and under these assumptions, the governing system of equations are given by:

$$
\nabla \cdot \mathbf{q}=0
$$

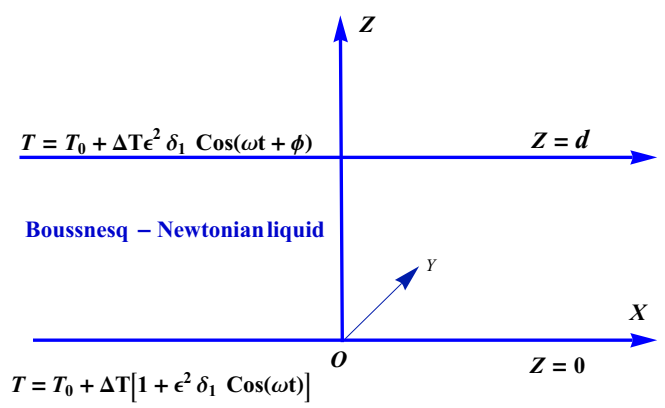

Fig .1 : Physical configuration for temperature modulation

$$
\begin{gathered}
\rho_{0}\left(\frac{\partial \mathbf{q}}{\partial t}+(\mathbf{q} . \nabla) \mathbf{q}\right)=-\nabla p-\rho \mathbf{g}+\mu(T) \nabla^{2} \mathbf{q}, \\
\frac{\partial T}{\partial t}+(\mathbf{q} . \nabla) T=\kappa_{T} \nabla^{2} T \\
\rho=\rho_{0}\left[1-\alpha_{T}\left(T-T_{0}\right)\right] \\
\mu(T)=\frac{\mu_{0}}{1+\epsilon^{2} \delta_{0}\left(T-T_{0}\right)}
\end{gathered}
$$

where $\mathbf{q}$ is velocity $(u, v, w), \mu(T)$ is a variable viscosity, $T$ is temperature, $\mathrm{p}$ is reduced pressure, $\kappa_{T}$ is the thermal diffusivity, $\alpha_{T}$ is thermal expansion coefficient, $\delta_{0}$ is small parameter indicating variation of viscosity with temperature, $\rho$ is the density, $\rho_{0}$ and $T_{0}$ are the reference density and temperature, $\epsilon$ is a quantity that indicates the smallness in order of magnitude of modulation and $t$ is the time. The externally imposed thermal boundary conditions are:

$$
\begin{aligned}
T & =T_{0}+\Delta T\left[1+\epsilon^{2} \delta_{1} \cos (\omega t)\right], & & \text { at } z=0 \\
& =T_{0}+\Delta T \epsilon^{2} \delta_{1} \cos (\omega t+\theta), & & \text { at } z=d
\end{aligned}
$$

where $\Delta T$ is the temperature gradient across the boundaries, $\delta_{1}, \omega$ are amplitude and frequency of temperature modulation, $\theta$ is the phase angle. The thermo-rhelogical relationship (5), is guided by Nield [28]. The basic state is assumed to be quiescent and the quantities in this state are given by

$$
q_{b}=0, \rho=\rho_{b}(z, t), p=p_{b}(z, t) \text { and } T=T_{b}(z, t) .
$$

Substituting Eq.(8), into Eqs.(1)-(3), we get the following relations which helps us to define basic state pressure and temperature:

$$
\begin{gathered}
\frac{d p_{b}}{d z}=-\rho_{b} \mathbf{g}, \\
\frac{\partial T_{b}}{\partial t}=\kappa_{T} \frac{\partial^{2} T_{b}}{\partial z^{2}}, \\
\rho_{b}=\rho_{0}\left[1-\alpha_{T}\left(T_{b}-T_{0}\right)\right] .
\end{gathered}
$$

The solution of equation (10), subjected to the boundary conditions (7), is given by:

$$
T_{b}(z, t)=T_{s}(z)+\epsilon^{2} \delta_{1} \operatorname{Re}\left[T_{1}(z, t)\right],
$$


where

$$
\begin{gathered}
T_{s}(z)=T_{0}+\Delta T\left(1-\frac{z}{d}\right), \\
T_{1}(z, t)=\left[a(\lambda) e^{\frac{\lambda z}{d}}+a(-\lambda) e^{\frac{-\lambda z}{d}}\right] e^{-i \omega t},
\end{gathered}
$$

and $a(\lambda)=\Delta T \frac{\left(e^{-i \theta}-e^{-\lambda}\right)}{\left(e^{\lambda}-e^{-\lambda}\right)}$ and $\lambda^{2}=\frac{-i \omega d^{2}}{\kappa_{T}}$.

Here $T_{s}(z)$ study part and $T_{1}(z, t)$ oscillatory part of basic state temperature $T_{b}(z, t)$. The finite amplitude perturbations on the basic state are superposed in the form:

$$
\mathbf{q}=\mathbf{q}_{b}+\mathbf{q}^{\prime}, \rho=\rho_{b}+\rho^{\prime}, p=p_{b}+p^{\prime}, T=T_{b}+T^{\prime} .
$$

Substituting Eq.(15), in Eqs.(1)-(3), and using the basic state results, we obtain:

$$
\nabla \cdot \mathbf{q}^{\prime}=0
$$

$$
\begin{gathered}
\rho_{0}\left(\frac{\partial \mathbf{q}^{\prime}}{\partial t}+\left(\mathbf{q}^{\prime} \cdot \nabla\right) \mathbf{q}^{\prime}\right)=-\nabla p+\alpha_{T} \rho_{0} \mathbf{g}(t) T^{\prime}+\mu(T) \nabla^{2} \mathbf{q}^{\prime}, \\
\frac{\partial T^{\prime}}{\partial t}+\mathbf{w}^{\prime} \frac{\partial T_{b}}{\partial z}+\left(\mathbf{q}^{\prime} \cdot \nabla\right) T^{\prime}=\kappa_{T} \nabla^{2} T^{\prime} .
\end{gathered}
$$

We consider only two-dimensional disturbances in our study, and hence introducing the stream function $\psi$ as $\mathbf{u}^{\prime}=$ $\frac{\partial \psi}{\partial z}, \mathbf{w}^{\prime}=-\frac{\partial \psi}{\partial x}$, We non-dimensionalize the physical variables as $(x, y, z)=d\left(x^{*}, y^{*}, z^{*}\right), t=\frac{d^{2}}{\kappa_{T}} t^{*}, \psi=\kappa_{T} \psi^{*}, T^{\prime}=$ $\Delta T T^{*}$ and $\omega=\frac{\kappa T}{d^{2}} \omega^{*}$. Now eliminating the pressure term and finally dropping the asterisk, we obtain:

$$
\begin{array}{r}
\frac{1}{\operatorname{Pr}} \frac{\partial}{\partial t}\left(\nabla^{2} \psi\right)-\frac{1}{\operatorname{Pr}} \frac{\partial\left(\psi, \nabla^{2} \psi\right)}{\partial(x, z)}=-R a_{T} \frac{\partial T}{\partial x} \\
+\bar{\mu}(T)\left(\nabla^{4} \psi\right)+\frac{\partial \bar{\mu}}{\partial z} \frac{\partial}{\partial z}\left(\nabla^{2} \psi\right), \\
-\frac{\partial T_{b}}{\partial z} \frac{\partial \psi}{\partial x}-\nabla^{2} T=-\frac{\partial T}{\partial t}+\frac{\partial(\psi, T)}{\partial(x, z)}
\end{array}
$$

where $\bar{\mu}(T)=\frac{1}{1+\epsilon^{2} V T}, \epsilon^{2}$ is a small quantity that indicates that the viscosity variation with temperature is weak. The non-dimensionalizing parameters in the above equations are: $\operatorname{Pr}=\frac{v}{\kappa_{T}}$ is Prandtl number, $R a_{T}=\frac{\alpha_{T} g \Delta T d^{3}}{v_{T}}$ is thermal Rayleigh number and $V=\delta_{0} \Delta T$ is thermo-rheological parameter or variable viscosity parameter, $v=\frac{\mu}{\rho_{0}}$ is kinematic viscosity. The basic state solution which appears in Eq.(20), influences the stability problem through the factor $\frac{\partial T_{b}}{\partial z}$, which is given by:

$$
\frac{\partial T_{b}}{\partial z}=-1+\epsilon^{2} \delta_{1}\left[f_{2}(z, t)\right]
$$

where

$$
f_{2}(z, t)=\operatorname{Re}\left[f(z) e^{(-i \omega t)}\right]
$$

$f(z)=\left[A(\lambda) e^{\lambda z}+A(-\lambda) e^{-\lambda z}\right]$

$A(\lambda)=\lambda \frac{\left(e^{-i \theta}-e^{-\lambda}\right)}{\left(e^{\lambda}-e^{-\lambda}\right)}$ and $\lambda=(1-i) \sqrt{\frac{\omega}{2}}$. We assume small variations of time, thus re-scaling it as $\tau=\epsilon^{2} t$, to study the stationary convection of the system. It is to be noted that over stable solutions are not considered in this problem. We write the non-linear Eqs.(19)-(20), in the matrix form as given bellow:

$$
\begin{gathered}
{\left[\begin{array}{cc}
-\bar{\mu}(T) \nabla^{4} & R a_{T} \frac{\partial}{\partial x} \\
\frac{\partial}{\partial x} & -\nabla^{2}
\end{array}\right]\left[\begin{array}{l}
\psi \\
T
\end{array}\right]=} \\
{\left[\begin{array}{c}
-\frac{\epsilon^{2}}{\operatorname{Pr}} \frac{\partial}{\partial \tau}\left(\nabla^{2} \psi\right)+\frac{1}{\operatorname{Pr}} \frac{\partial\left(\psi, \nabla^{2} \psi\right)}{\partial(x, z)}+\frac{\partial \bar{\mu}}{\partial z} \frac{\partial}{\partial z}\left(\nabla^{2} \psi\right) \\
-\epsilon^{2} \frac{\partial T}{\partial \tau}+\frac{\partial(\psi, T)}{\partial(x, z)}+\epsilon^{2} \delta_{1} f_{2}(z, \tau) \frac{\partial \psi}{\partial x}
\end{array}\right] .}
\end{gathered}
$$

We solve Eq.(23), by using $\bar{\mu}(T)=\bar{\mu}\left(T_{b}\right)$ [28], and considering stress free and isothermal boundary conditions as given bellow:

$$
\psi=T=0 \text { at } z=0 \& z=1 .
$$

\section{Ginzburg-Landau equation and heat transport}

We introduce the following asymptotic expansions in Eq.(23):

$$
\begin{gathered}
R a_{T}=R_{0 c}+\epsilon^{2} R_{2}+\epsilon^{4} R_{4}+\ldots, \\
\psi=\epsilon \psi_{1}+\epsilon^{2} \psi_{2}+\epsilon^{3} \psi_{3}+\ldots, \\
T=\epsilon T_{1}+\epsilon^{2} T_{2}+\epsilon^{3} T_{3}+\ldots,
\end{gathered}
$$

where $R_{0 c}$ is the critical value of the Rayleigh number at which the onset of convection takes place in the absence of temperature modulation. Now we solve the system for different orders of $\epsilon$.

At the lowest order, we have:

$$
\left[\begin{array}{cc}
-\nabla^{4} & R_{0 c} \frac{\partial}{\partial x} \\
\frac{\partial}{\partial x} & -\nabla^{2}
\end{array}\right]\left[\begin{array}{l}
\psi_{1} \\
T_{1}
\end{array}\right]=\left[\begin{array}{l}
0 \\
0
\end{array}\right] .
$$

The solution of the lowest order system subject to the boundary conditions Eq.(24), is:

$$
\begin{aligned}
& \psi_{1}=A(\tau) \sin \left(k_{c} x\right) \sin (\pi z), \\
& T_{1}=-\frac{k_{c}}{\delta^{2}} A(\tau) \cos \left(k_{c} x\right) \sin (\pi z),
\end{aligned}
$$

where $\delta^{2}=k_{c}^{2}+\pi^{2}$. The critical value of the Rayleigh number and the corresponding wave number for the onset of stationary convection is calculated numerically and the expression are given by:

$$
\begin{aligned}
& R_{0 c}=\frac{\delta^{6}}{k_{c}^{2}}, \\
& k_{c}=\frac{\pi}{\sqrt{2}},
\end{aligned}
$$

which are the results given by Chandrasekhar [1]. At the second order, we have:

$$
\left[\begin{array}{cc}
-\nabla^{4} & R_{0 c} \frac{\partial}{\partial x} \\
\frac{\partial}{\partial x} & -\nabla^{2}
\end{array}\right]\left[\begin{array}{l}
\psi_{2} \\
T_{2}
\end{array}\right]=\left[\begin{array}{l}
R_{21} \\
R_{22}
\end{array}\right],
$$

where

$$
\begin{aligned}
& R_{21}=0 \\
& R_{22}=\frac{\partial \psi_{1}}{\partial x} \frac{\partial T_{1}}{\partial z}-\frac{\partial \psi_{1}}{\partial z} \frac{\partial T_{1}}{\partial x}
\end{aligned}
$$


The second order solutions subjected to the boundary conditions Eq.(24), is obtained as follows:

$$
\begin{aligned}
& \psi_{2}=0, \\
& T_{2}=-\frac{k_{c}^{2}}{8 \pi \delta^{2}} A^{2}(\tau) \sin (2 \pi z) .
\end{aligned}
$$

The horizontally averaged Nusselt number, $N u(\tau)$, for the stationary mode of convection is given by:

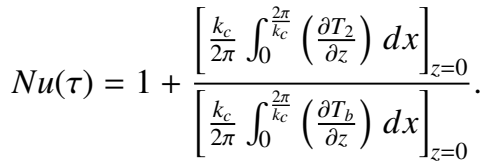

It is clear that the temperature modulation is effective at $O\left(\epsilon^{2}\right)$ and affects $N u(\tau)$ through $A(\tau)$ which is obtained in the third order solution. Substituting $T_{2}$ and $\frac{\partial T_{b}}{\partial z}$ value from Eq.(21), in Eq.(38), and simplifying we get:

$$
N u(\tau)=1+\frac{k_{c}^{2}}{4 \delta^{2}} A^{2}(\tau)
$$

At the third order, we have:

$$
\left[\begin{array}{cc}
-\nabla^{4} & R_{0 c} \frac{\partial}{\partial x} \\
\frac{\partial}{\partial x} & -\nabla^{2}
\end{array}\right]\left[\begin{array}{l}
\psi_{3} \\
T_{3}
\end{array}\right]=\left[\begin{array}{l}
R_{31} \\
R_{32}
\end{array}\right],
$$

where

$$
\begin{gathered}
R_{31}=-\frac{1}{\operatorname{Pr}} \frac{\partial}{\partial \tau}\left(\nabla^{2} \psi_{1}\right)+V T_{b} \nabla^{4} \psi_{1}+V \frac{\partial}{\partial z}\left(\nabla^{2} \psi_{1}\right) \\
-2 R_{0 c} V T_{b} \frac{\partial T_{1}}{\partial x}-R_{2} \frac{\partial T_{1}}{\partial x}, \\
R_{32}=\frac{\partial \psi_{1}}{\partial x} \frac{\partial T 2}{\partial z}+\delta_{1} f_{2}(z, \tau) \frac{\partial \psi_{1}}{\partial x}-\frac{\partial T_{1}}{\partial \tau} .
\end{gathered}
$$

Substituting $\psi_{1}, T_{1}$ and $T_{2}$ into Eqs.(42)-(43), we obtain expressions for $R_{31}$ and $R_{32}$ easily. Now, we apply the solvability condition for the existence of third order solution, and get the Ginzburg-Landau equation for the stationary mode of convection with time-periodic coefficients in the form:

$$
A_{1} A^{\prime}(\tau)-A_{2} A(\tau)+A_{3} A(\tau)^{3}=0,
$$

where $A_{1}=\frac{1}{\delta^{2}}\left[\frac{(1+P r)}{P r}\right], A_{2}=\left[\frac{R_{2}}{R_{0 c}}+\frac{V}{2}-2 \delta_{1} I_{1}\right], A_{3}=\frac{k_{c}^{2}}{8 \delta^{2}}$ and

$$
I_{1}=\int_{0}^{1} f_{2}(z, \tau) \sin ^{2}(\pi z) d z .
$$

\section{Analytical solution for Unmodulated case}

In the case of unmodulated fluid layer, the above Ginzburg Landau equation can be written as:

$$
A_{1} A_{u}^{\prime}(\tau)-A_{2} A_{u}(\tau)+A_{3} A_{u}(\tau)^{3}=0,
$$

where $A_{u}(\tau)$ is an amplitude of convection for unmodulated case and $A_{1}, A_{3}$ have the same expression as given in the
Eq.(44), and $A_{2}=1+\frac{V}{2}$. The solution of Eq.(45), is given by:

$$
A_{u}(\tau)=\frac{1}{\sqrt{\left(\frac{A_{3}}{2 A_{2}}+C_{1} \operatorname{Exp}\left[\frac{-2 A_{2}}{A_{1}}\right]\right)}},
$$

where $C_{1}$ is a parameter, which can be calculated for a given suitable initial condition. The horizontal averaged Nusselt number in this case is obtained from Eq.(39), by using the value of $A_{u}(\tau)$ in the place of $A(\tau)$.

\section{Results and discussion}

The problem addresses non-linear stability of Rayleigh Bénard convection in a temperature-dependent viscosity liquid under temperature modulation effect. The temperature sensitivity of the fluid is modeled through a thermorheological relationship that was proposed by Nield [28]. Here we have presented a weakly nonlinear stability theory to investigate the effect of temperature modulation and thermo-rheological parameter on heat transport. The effect of temperature modulation on Rayleigh Bénard system has been assumed to be of order $O\left(\epsilon^{2}\right)$ which shows that, we consider only small amplitude of temperature modulation. This assumption will help us in obtaining the corresponding amplitude equations in a simpler and easier manner than in Lorenz model. The parameters that arise in the problem are $\operatorname{Pr}, V, \theta, \delta_{1}$ and $\omega$, and these parameters influence the convective heat transport. The first two parameters relate to the fluid layer and the last three concern the external mechanism of controlling convection. The fluid layer is not considered to be highly viscous, therefore only moderate value of $\mathrm{Pr}$ are taken for calculations. Because small amplitude modulations are considered, the values of $\delta_{1}$ lie between 0 and 0.5 . Further, the modulation of the boundary temperature is assumed to be of low frequency. At low range of frequencies, the effect of frequencies on onset of convection as well as on heat transport is maximum. The values of thermo-rheological parameter $V$ also considered to be small. Nusselt number $N u$ with respect to time $\tau$ has been depicted in Figs.(2-4). The effect of temperature modulation has been shown in Figs.(2-4), while in Fig.(4) we have shown comparison between various cases of temperature modulation. The temperature modulation of the boundaries has been considered in the following three cases:

1. In-phase modulation (IPM) $(\theta=0)$,

2. Out-phase modulation (OPM) $(\theta=\pi)$ and

3. Lower-boundary modulated only (LBMO) $(\theta=-i \infty)$. which means that only lower boundary temperature is modulated, the upper boundary is kept at fixed constant temperature.

From the figures we observe that, the value of Nusselt number starts with 1 , thus showing the conduction state initially. Then, there is a sudden increase in its values for intermediate values of time $\tau$, showing that convection is taking place. Finally, when $\tau$ is large, $N u$ approaches fixed values in all three respective cases, thus showing that the steady state has been achieved. However, the value of $\mathrm{Nu}$ becomes oscillatory at large and intermediate value of time $\tau$ for OPM and LBMO cases. 
In - Phase Modulation $(\phi=0)$
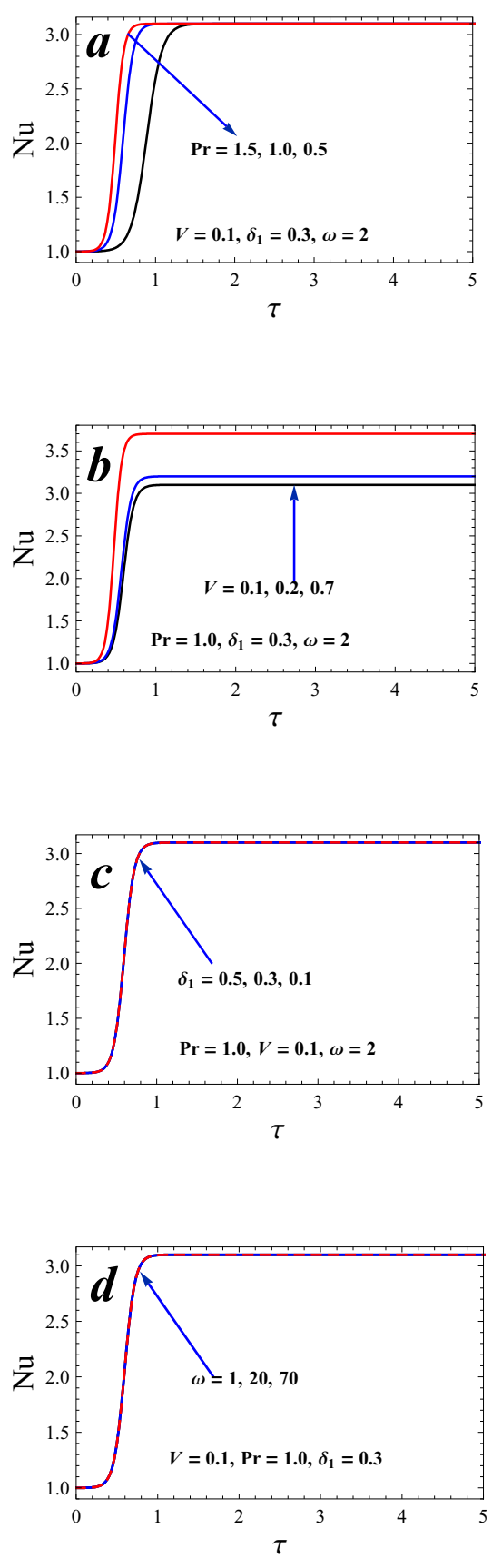

Fig. 2 : Nu vns $\tau$ for (a) $\operatorname{Pr}(\mathrm{b}) \mathrm{V}$ (c) $\delta_{1}$ (d) $\omega$

In Fig.(2) we depict variation of $N u$ with respect to time $\tau$ for in phase modulation. We find from the Fig.(2a,b) that, $N u$ increases on increasing $P r$, showing that, the effect of an increment in $P r$ is to increase the heat transfer by advancing the onset of convection. Similar effect is also found for the thermo-rheological parameter $V$. Further, from the Figs.(2c-d), we observed that on increasing the values of amplitude $\delta_{1}$ and frequency $\omega$ of modulation, the value of Nusselt number $N u(\tau)$ does not alter. This shows that increments in $\delta_{1}$ and $\omega$ have negligible effect on rate of heat transfer. Also, in case of in-phase modulation, we obtain qualitatively similar results to that of unmodulated case, which may be due to the fact that in-phase tem-
Out of Phase Modulation $(\phi=\pi)$
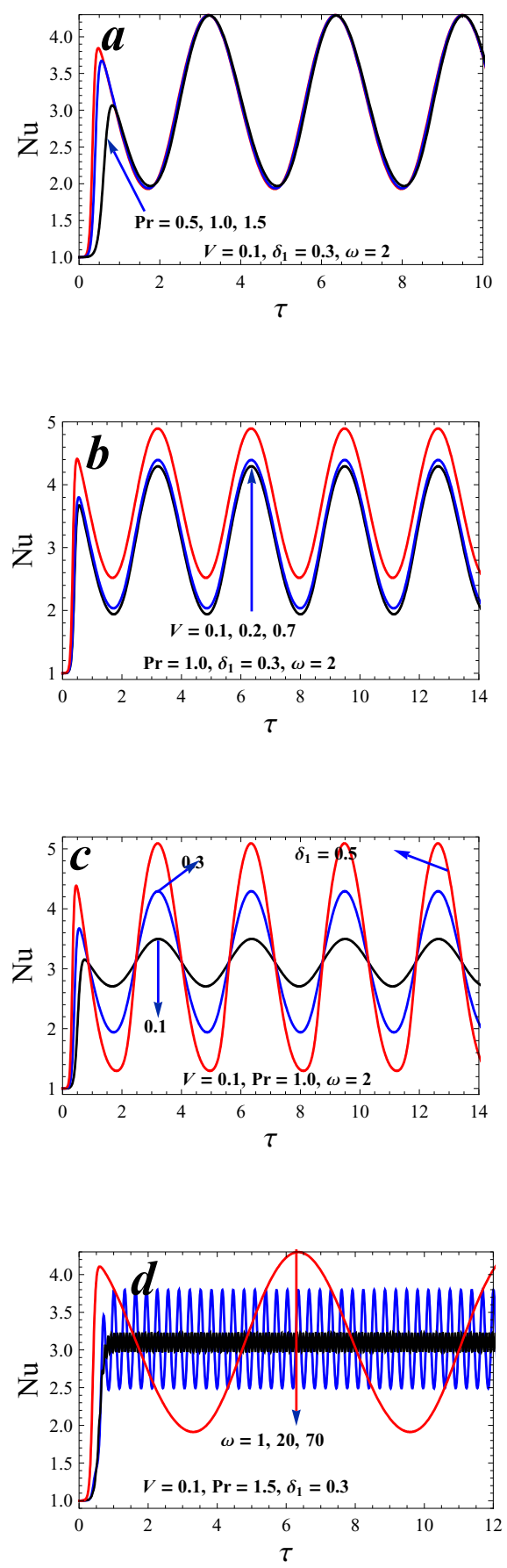

Fig. $3:$ Nu vns $\tau$ for (a) $\operatorname{Pr}(\mathrm{b}) \mathrm{V}$ (c) $\delta_{1}$ (d) $\omega$

perature modulation of the boundaries hardly makes any change in the temperature gradient across the fluid layer, therefore the results are same as that of unmodulated case.

Fig.3, shows the plots of $N u$ with time $\tau$ for the case of OPM. Figs.(3a-b), displays the effect of $\mathrm{Pr}$ and $\mathrm{V}$ on $\mathrm{Nu}$. From the figures, we find that on increasing $\mathrm{Pr}$ and $\mathrm{V}, \mathrm{Nu}$ increases, thus the effect is destabilizing as in IPM. From Fig. $3 \mathrm{c}$, we find that the effect of an increment in modulation amplitude $\delta_{1}$ on $N u$ is to increase the magnitude of $N u$, i.e., increasing the rate of heat transport. From Fig.3d, we observe that on increasing the frequency of modulation, the 


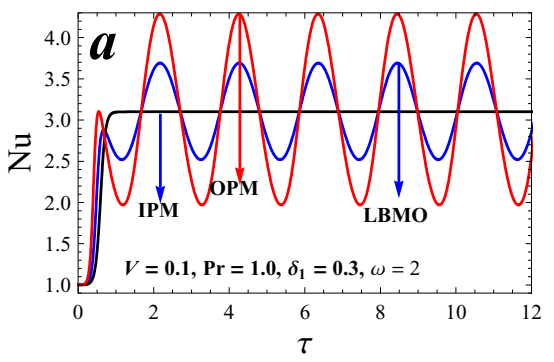

Fig . 4 : Comparisionof different temperatureprofiles.

magnitude of $\mathrm{Nu}$ decreases, and shortens the wavelength of oscillations. As the frequency increases from 1 to 70 , the magnitude on $N u$ is decreases, and so the effect of temperature modulation on heat transport diminishes. On further increasing the value of $\omega$, the effect of temperature modulation on thermal instability disappears altogether. Thus at high frequency of modulation, the present results confirm the results of Venezian [3], and Bhadauria and Bhatia [12].

It was found that, for lower boundary temperature modulation the results are similar to those in OPM case given in Figs.(3a-d), however, for avoiding repetition of similar figures we have omitted inclusion of figures for LBMO case.

In Fig.4, we compared the results of all three types of temperature modulation. We observe that in general:

$$
N u_{I P M}<N u_{L B M O}<N u_{O P M} .
$$

In the case of unmodulated system, we found amplitude of convection analytically given by Eq.(46), and obtained Nusselt number, depicted plot's $N u$ verses $\tau$. It is found that, in unmodulated case, it is exactly the same as in inphase temperature modulation.

\section{Conclusions}

The effect of temperature modulation on thermal instability in a temperature dependent viscous fluid layer has been investigated by performing a weakly nonlinear stability analysis. The Ginzburg-Landau amplitude equation has been obtained for an amplitude of convection in the case of stationary mode of thermal instability. The following conclusions are drawn from the above analysis:

1. Temperature modulation can be used as an external means to augment/diminish heat transport in a fluid layer.

2. The IPM is negligible on heat transport.

3. The effect of increasing Prandtl number $P r$ is to advance the convection in all three cases of modulation.

4. Effect of thermo-rhelogical parameter $V$ is to enhance the heat transport in all three types of modulation.

5. An increment in magnitude and frequency of modulation has negligible effect on heat trasport in IPM case, while there is significant effect in the cases of OPM and LBMO.

6. In the case of OPM and LBMO, $N u(\tau)$ shows an oscillatory nature.

7. As time $\tau$ increases, the magnitude of streamlines increases and isotherms loses their evenness, showing that convection is taking place. At $\tau=1.0$ the system achieves steady state.
8. The thermo-rheological model of Nield [28], gives physically acceptable results, namely, the destabilizing effect of variable viscosity on Rayleigh-Bénard convection and thereby an enhanced heat transport.

\section{ACKNOWLEDGMENT}

This work was done during the leave sanctioned to the author by Banaras Hindu University, Varanasi, India to work as professor of Mathematics at Department of Applied Mathematics, School for Physical Sciences, Babasaheb Bhimrao Ambedkar central University, Lucknow, India. Author gratefully acknowledges Banaras Hindu University, Varanasi, India for the same.

\section{References}

1. S. Chandrasekhar, "Hydrodynamic and Hydromagnetic stability,'Oxford University Press London, (1961).

2. P.G. Drazin, D.H. Reid, "Hydrodynamic stability,"Cambridge University Press Cambridge, (2004).

3. G. Venezian, "Effect of modulation on the onset of thermal convection,'J. Fluid. Mech., 35, 243-254, (1969).

4. R.J. Donnelly, "Experiments on the stability of viscous flow between rotating cylinders III: enhancement of hydrodynamic stability by modulation,"Proc. R. Soc. Lond. Ser., A, 281, 130-139, (1964).

5. S. Rosenblat, D.M. Herbert, "Low frequency modulation of thermal instability,”J. Fluid. Mech., 385-389, (1970).

6. S. Rosenblat, G.A. Tanaka, "Modulation of thermal convection instability,'Phys. Fluids., 14(7), 1319-1322, (1971).

7. C.S. Yih, C.H. Li, "Instability of unsteady flows or configurations, Part 2. Convective instability,"J. Fluid. Mech., 54, 143, (1972).

8. K. Kumar, J.K. Bhattacharjee, K. Banerjee, "Onset of the first instability in hydrodynamic flows: effect of parametric modulation,"Phys. Rev., A. 34, 5000-5006, (1986).

9. R.G. Finucane, R.E. Kelly, "Onset of instability in afluid layer heated sinusoidally from bellow,'Int. J. Heat. Mass. Transf., 19, 71-85, (1976).

10. M.H. Roppo, S.H. Davis, S. Rosenblat, "Bénard convection with time periodic heating,"Phys. Fluids., 27, 796-803, (1984).

11. P.K. Bhatia, B.S. Bhadauria, "Effect of modulation on thermal convection instability,"Z. Naturforsch., 55a, 957-966, (2000).

12. B.S. Bhadauria, P.K. Bhatia, "Time periodic heating of Rayleigh-Bénard convection,’Physica. Scripta., 66, 59-65, (2002).

13. B.S. Bhadauria, "Temperature modulation of double diffusive convection in a horizontal fluid layer,' $Z$. Naturforsch., 61a, 335-344, (2006).

14. B.S. Bhadauria, "Time-periodic heating of Rayleigh-Bénard convection in a vertical magnetic field,'Physica. Scripta., 73(3), (2006). 296-302.

15. M.S. Malashetty, M. Swamy, "Efect of thrmal modulation on the onset of convection in rotating fluid ayer,'Int. J. heat. mass. transp., 51, 2814-2823, (2008) 
16. B.S. Bhadauria, P.K. Bhatia, L. Debnath, "Weakly non-linear analysis of Rayleigh-Bénard convection with time periodic heating,'Int. J. Non-Linear. Mech. 44(1), 58-65, (2009).

17. V.R.K. Raju, S.N. Bhattacharya, "Onset of thermal instability in a horizontal layer of fluid with modulated boundary temperatures,"J. Engg. Math., 66, 343-351, (2010).

18. P.G. Siddheshwar, B.S. Bhadauria, Pankaj Mishra, A.K. Srivastava, "Study of heat transport by stationary magneto convection in a Newtonian liquid under temperature or gravity modulation using GinzburgLandau model,'Int. J. non-linear. Mech. 47, 418-425, (2012).

19. B.S. Bhadauria, P.G. Siddheshwar, Om.P. Suthar, "Non-linear thermal instability in rotating viscous fluid layer under temperature/gravity modulation,”ASME. J. heat. transf., 34, 102502, (2012).

20. B.S. Bhadauria, I. Hashim, P.G. Siddheshwar, "Effects of Time-Periodic Thermal Boundary Conditions and Internal Heating on Heat Transport in a Porous Medium,'Transp. Porous. Media., 97, 185-200, (2013).

21. X.Z. Wu, A. Libchaber, "Non-Boussinesq effects in free thermal convection,"Phys. Rev., A43, 2833-2839, (1991).

22. N.G. Kafoussius, E.M. Williams, "The effect of temperature-dependent viscosity on the free convective laminar boundary layer flow past a vertical isothermal plate,"Acta. Mech., 110, 123-137, (1995).

23. N.G. Kafoussius, D.A.S. Rees, "Numerical study of the combined free and forced convective laminar boundary layer flow past a vertical isothermal flat plate with temperature-dependent viscosity,"Acta. Mech., 127, 39-50, (1998).

24. M.M. Molla, M.A. Hossain, R.S.R. Gorla, "Natural convection flow from an isothermal circular cylinder with temperature-dependent viscosity,"Heat. Mass. Transf., 41, 594-598 (2005).

25. D. Pal, H. Mondal, "Influence of temperaturedependent viscosity and thermal radiation on MHD-forced convection over a non-isothermal wedge,"Appl. Math. Comput., 212, 194-208, (2009).

26. Ching-Yang Cheng, "Natural convection boundary layer flow of fluid with temperature-dependent viscosity from a horizontal elliptical cylinder with constant surface heat flux,"Appl. Math. Comput., 217, 83-91, (2010).

27. S. Nadeem, N.S Akbar, "Effects of temperature dependent viscosity on peristaltic flow of a Jeffrey-six constant fluid in a non-uniform vertical tube,"Commun. Nonlinear. Sci. Numer. Simul., 15, 3950, (2010).

28. D.A. Nield, "The effect of temperature-dependent viscosity on the onset of convection in a saturated porous medium,"ASME. J. Heat. Transf., 118, 803-805, (1996).

29. B.S. Bhadauria, Palle. Kiran, "Heat transport in an anisotropic porous medium saturated with variable viscosity liquid under temperature modulation,'Transp. Porous. Media., 100, 279-295, (2013). 